\title{
An integrated torque-vectoring control framework for electric vehicles featuring multiple handling and energy- efficiency modes selectable by the driver
}

\author{
Andrea Mangia $\cdot$ Basilio Lenzo $(\mathbb{D} \cdot$ Edoardo Sabbioni
}

Received: 20 April 2020/Accepted: 29 January 2021

(C) The Author(s) 2021

\begin{abstract}
A key feature achievable by electric vehicles with multiple motors is torque-vectoring. Many control techniques have been developed to harness torque-vectoring in order to improve vehicle safety and energy efficiency. The majority of the existing contributions only deal with specific aspects of torque-vectoring. This paper presents an integrated approach allowing a smooth coordination among the main blocks that constitute a torque-vectoring control framework: (1) a reference generator, that defines target yaw rate and sideslip angle; (2) a high level controller, that works out the required total torque and yaw moment at the vehicle level; (3) a low level controller, that maps the required force and yaw moment into individual wheel torque demands. In this framework, the driver can select one among a number of driving modes that allow to change the vehicle cornering response and, as a second priority, maximise energy efficiency. For the first time, the
\end{abstract}

A. Mangia · B. Lenzo ( $\square)$

Department of Engineering and Mathematics, Sheffield

Hallam University, Sheffield, UK

e-mail: basilio.lenzo@shu.ac.uk

\section{B. Lenzo}

Department of Industrial Engineering, University of

Padova, Padua, Italy

E. Sabbioni

Department of Mechanical Engineering, Politecnico di

Milano, Milan, Italy selectable driving modes include an "Energy efficiency" mode that uses torque-vectoring to prioritise the maximisation of the vehicle energy efficiency, thus further increasing the vehicle driving range. Simulation results show the effectiveness of the proposed framework on an experimentally validated 14 degrees of freedom vehicle model.

Keywords Torque-vectoring - Direct yaw moment . Electric vehicles · Energy efficiency · Driving modes

\section{List of symbols}

A Dynamic matrix of the state-space representation of the vehicle dynamics

a Vehicle front semi-wheelbase

$a_{x} \quad$ Longitudinal acceleration

$a_{y} \quad$ Lateral acceleration

$a_{y}^{*} \quad$ Lateral acceleration limit for the linear region of desired cornering response

$a_{y, M A X} \quad$ Maximum lateral acceleration for the desired cornering response

$B_{M_{z}} \quad$ Input matrix of the state-space representation of the vehicle dynamics

$B_{\delta} \quad$ Disturbance input matrix of the state-space representation of the vehicle dynamics

$C_{i} \quad$ Cornering stiffness of axle $i$

$c_{1} \quad$ Coefficient used in the definition of $f$

$c_{2} \quad$ Coefficient used in the definition of $f$

$D \quad$ Maximum achievable tangential force according to Pacejka's Magic Formula 
$d_{1} \quad$ Coefficient of Pacejka's Magic Formula (linear dependence on $F_{z, i j}$ )

$d_{2} \quad$ Coefficient of Pacejka's Magic Formula (nonlinear dependence on $F_{z, i j}$ )

$E_{\text {loss }, \text { tot }}$ Total energy loss during the mild slalom

$e \quad$ Error vector

$F_{x, i j} \quad$ Longitudinal force on wheel $i j$

$F_{y, i j} \quad$ Lateral force on wheel $i j$

$F_{y, i j}^{M A X}$

$F_{t o t, i j}^{M A X}$

$F_{z, 0} \quad$ Coefficient of Pacejka's Magic Formula

$F_{z, i j} \quad$ Vertical force on wheel $i j$

$F_{z, s t a t, i j} \quad$ Static load on wheel $i j$

$f \quad$ Function of $I_{Y}$

$G \quad$ Matrix obtained through the solution of a

Riccati equation for the minimisation of $J$

$g \quad$ Gravitational acceleration

$h \quad$ Height of the vehicle centre of mass

$I_{Y} \quad$ Yaw index

$i \quad$ Subscript indicating vehicle axle $(i=1$

front axle, $i=2$ rear axle)

$J \quad$ Performance index to be minimised in the high level controller

$J_{z} \quad$ Vehicle moment of inertia with respect to a vertical axis through the centre of mass

$j \quad$ Subscript indicating vehicle side $(j=1$ left side, $j=2$ right side)

$K_{u s, b} \quad$ Understeer coefficient for the baseline vehicle

$K_{u s} \quad$ Understeer coefficient for the desired cornering response

$k_{n p} \quad$ Coefficient of a third order polynomial approximation of $P_{l o s s, i j}$

$l \quad$ Vehicle wheelbase

$M_{z} \quad$ Desired yaw moment

$M_{z, M A X} \quad$ Maximum achievable yaw moment

$M_{z, S S C} \quad$ Optimal yaw moment contribution for steady-state conditions

$M_{z, T C} \quad$ Stability yaw moment contribution for transient conditions

$m \quad$ Vehicle mass

$N_{r} \quad$ Stability derivative multiplying $r$ in the rotational equilibrium equation

$N_{\beta} \quad$ Stability derivative multiplying $\beta$ in the rotational equilibrium equation

$n$
$P_{b} \quad$ Normalising factor for the motor power loss in the third order polynomial approximation of $P_{l o s s, i j}$

$P_{\text {in }} \quad$ Motor input power

$P_{\text {loss }, i j} \quad$ Power losses for motor $i j$

$P_{l o s s, x, i j} \quad$ Longitudinal tyre slip power losses at wheel $i j$

$P_{l o s s, y, i j} \quad$ Lateral tyre slip power losses at wheel $i j$

$P_{\text {out }} \quad$ Motor output power

$p \quad$ Subscript used in the definition of $k_{n p}$

$Q \quad$ Matrix accounting for $e$ in the definition of $J$

$q \quad$ Subscript referring to the external side of the vehicle

$R \quad$ Matrix accounting for $M_{z}$ in the definition of $J$

$R_{c} \quad$ Radius of curvature of the vehicle trajectory

$R_{w} \quad$ Average wheel radius

$r \quad$ Vehicle yaw rate

$\dot{r} \quad$ Time derivative of $r$

$r_{M A X} \quad$ Maximum yaw rate used for the definition of $Q$

$r_{\text {ref }} \quad$ Reference yaw rate

$r_{r e f, S}$

$T_{i j} \quad$ Torque demand at wheel $i j$

$T_{\text {max } \text { side }}$ Maximum torque for the vehicle side

$T_{L} \quad$ Torque demand for the left vehicle side

$T_{R} \quad$ Torque demand for the right vehicle side

$T_{s w} \quad$ Switching torque for a vehicle side

$T_{\text {tot }} \quad$ Desired total torque

$V \quad$ Vehicle velocity

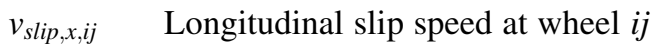

$v_{s l i p, y, i j} \quad$ Lateral slip speed at wheel $i j$

$w \quad$ Vehicle track width

$x \quad$ State vector

$\dot{x} \quad$ Time derivative of $x$

$x_{i} \quad$ Lateral load transfer distribution coefficient between front and rear axle

$Y_{r} \quad$ Stability derivative multiplying $r$ in the translational equilibrium equation

$Y_{\beta} \quad$ Stability derivative multiplying $\beta$ in the translational equilibrium equation

$\beta \quad$ Vehicle sideslip angle

$\dot{\beta} \quad$ Time derivative of $\beta$

$\beta_{\text {MAX }} \quad$ Maximum sideslip angle used for the definition of $\beta_{\text {ref }}$ 
$\beta_{\text {ref }} \quad$ Reference sideslip angle

$\Gamma_{b} \quad$ Normalising factor for the motor torque in the third order polynomial approximation of $P_{\text {loss }, i j}$

$\Gamma_{\max , i j} \quad$ Maximum torque for motor $i j$ based on the motor characteristics

$\Delta E_{\text {loss,tot }}$ Total energy loss variation with respect to the baseline vehicle for the mild slalom

$\Delta P_{\text {loss,tot }}$ Total power loss variation with respect to the baseline vehicle for the ramp steer

$\Delta T_{L R} \quad$ Torque unbalance between left and right vehicle side

$\delta \quad$ Wheel steer angle

$\delta_{d y n} \quad$ Dynamic steering wheel angle

$\delta_{k i n} \quad$ Kinematic steering wheel angle

$\delta_{s w} \quad$ Steering wheel angle

$\delta_{s w, t h} \quad$ Threshold for $\delta_{s w}$ for the Energy efficiency mode

$\eta_{i j} \quad$ Efficiency of motor $i j$

$\mu \quad$ Estimated tyre-road friction coefficient

$\sigma \quad$ Generic front-to-total wheel torque distribution factor

$\sigma_{L} \quad$ Front-to-total wheel torque distribution factor for the left vehicle side

$\sigma_{R} \quad$ Front-to-total wheel torque distribution factor for the right vehicle side

$\tau \quad$ Steering ratio

$\Omega_{b} \quad$ Normalising factor for the motor speed in the third order polynomial approximation of $P_{\text {loss }, i j}$

$\Omega_{i j} \quad$ Angular speed of motor $i j$

$\omega_{i j} \quad$ Angular speed of wheel $i j$

\section{Introduction}

Recent years have seen an increasingly large interest in vehicle electrification and Advanced Driver Assistance Systems (ADAS), both deemed key features for the future of private transportation. From an engineering point of view, vehicle electrification has opened plenty of possibilities in terms of powertrain layouts and exploitable features, some of which are simply impossible to achieve with a traditional architecture [1-3].

A very interesting layout for electric vehicles consists of either four motors (one per wheel) or two motors on the same axle, i.e., one on the left wheel and one on the right wheel of the axle [4]. In both cases, no mechanical differential is present, and two main configurations are possible: (1) in-wheel motors, where motors are installed within wheel hubs; (2) on-board motors, or "close-to-wheel", which require a mechanical transmission to reach the wheels. The possibility of allocating desired amounts of torque to each motor is known as torque-vectoring (TV), a key feature for vehicle control. In fact, by allocating different amounts of torque on the left and right hand sides of the vehicle, a direct yaw moment can be generated hence exploited to control the vehicle behaviour. Notably, also a front-rear torque bias can influence the vehicle behaviour, even if with much less potential than direct yaw moment control [5].

Different TV control algorithms can be employed, depending on specific vehicle performance requirements. Typical examples of desirable effects include the improvement of safety, stability and cornering performance $[6,7]$, or the enhancement of energy efficiency $[8,9]$. Also ADAS provide safety benefits, but with important differences. A notable example of ADAS is the Electronic Stability Control which, when an emergency condition is detected, intervenes on individual braking torques in order to generate a correcting yaw moment. Conversely, TV is continuously active, which further benefits vehicle safety and stability [10]. Other interesting ADAS are steering control systems, which are being increasingly adopted in modern cars: notable examples are the Lane Keeping System, or the Automatic Emergency Steering $[11,12]$. Their principle of operation is quite different than TV as they directly intervene on the steering wheel angle position in particular situations, such as driver distractions. Some literature studies propose to combine the effect of direct yaw moment and steering control systems [13].

While direct yaw moment control may also be achieved in traditional cars (e.g. with an internal combustion engine) through appropriate mechanical arrangements [14], such as a limited-slip differential, these systems present important limitations, e.g. the impossibility to allocate more torque on the faster wheel of the axle. Instead, an electric vehicle layout with multiple motors and no mechanical differential allows complete freedom in this sense, and it will be the target of the approach presented in this paper. 
The majority of the literature agree that a TV control algorithm is composed by three main blocks:

- Reference generator

- High level controller

- Low level controller

(a) Reference generator

The reference generator combines information on measured/estimated vehicle states and on the driver input, thereby generating reference values for relevant vehicle quantities, e.g. a reference yaw rate. Such references are to be followed by the vehicle through TV. Most reference yaw rate generators are based on a simple principle, i.e. tuning the understeer coefficient $K_{u s}$ [5, 6, 15-18].

An interesting approach was proposed in [19] and then adopted by many other contributions. The idea is to design the whole understeer characteristic of the vehicle, which can potentially follow any desired profile and not necessarily a simple linear behaviour - which is the case when the reference yaw rate is based on tuning the sole understeer coefficient. In particular, a piecewise function is imposed as the relationship between dynamic steering angle and lateral acceleration. This allows to:

1. Reduce the understeer gradient with respect to the baseline vehicle (i.e. the same vehicle plant without TV controller)

2. Extend the region of linear cornering response

3. Increase the maximum lateral acceleration achievable with the available tyre-road friction conditions

By designing multiple understeer characteristics, different vehicle behaviours can be obtained, denoted as driving modes [20]. Different driving modes can be selected by the driver to adapt the vehicles behaviour as desired, e.g. improving the fun-to-drive, adapt to low friction conditions, etc.

Another target quantity often taken into account is the vehicle sideslip angle. Studies in the literature deal with it in two ways: either by defining a reference sideslip angle and integrating such requirement together with the reference yaw rate [18], or by correcting the reference yaw rate based on the actual value of sideslip angle and appropriate thresholds [21]. In any case this represents an important challenge since controlling yaw rate and sideslip angle at the same time often means to deal with contrasting requirements. That implies the need for the controller to be carefully tuned so as to appropriately mediate between the two requirements while guaranteeing safety and stability for the vehicle.

A limitation of most researches in the scientific literature is that driving modes are designed taking into account only vehicle handling requirements, but not energy efficiency requirements. Few works look at the design of understeer characteristics for improving energy efficiency [22, 23] but no efforts have been made so far to propose an integrated approach which allows either targets depending on the driver preferences/priorities.

(b) High level controller

The high level controller takes the output target quantities from the reference generator, as well as the driver input (e.g. accelerator/brake pedal positions) and vehicle states (e.g. vehicle velocity). Such inputs are elaborated to work out the desired total torque $T_{\text {tot }}$ and yaw moment $M_{z}$ to be applied at the vehicle level. In terms of total torque demand, that is often achieved through a driveability map that takes the vehicle speed as input [10]. For the yaw moment, the simplest method is the implementation of a ProportionalIntegral-Derivative (PID) controller based on the error between reference and actual value (e.g. of the yaw rate). Other options include e.g. $\mathrm{H}-\infty$ control [24] and sliding mode control [25-27]. In [18] the problem is dealt by using a Linear Quadratic Regulator (LQR) and introducing a parameter denoted as yaw index that accounts for the rate of change of the sideslip angle. Interesting comparisons of feedback control techniques are presented in [28, 29].

(c) Low level controller

The task of the low level controller is to map the desired total torque and yaw moment into values of torque demand for each motor. Considering a 
typical electric vehicle layout featuring four motors, there are potentially four degrees of freedom (DOF), i.e. the four values of torque that can be assigned. Since the total torque and the yaw moment are imposed, practically there are only two DOF, i.e. the desired total torque and yaw moment can be achieved with virtually $\infty^{2}$ combinations of the four motor torques. Dizqah et al. [30] show that the desired total torque and yaw moment demands at the vehicle level can be equivalently seen as two values of torque demand, one per each side of the vehicle. Therefore, the available two DOF can be seen as distribution factors between front and rear axle, one per side. Such factors can be determined based on energy efficiency criteria. Because of this, and since the yaw moment accounts for handling requirements, the objectives considered in the TV controller definition can be multiple. Notable examples are in [27, 31].

On the other hand, energy efficiency might be the only target, in which case the yaw moment would not be imposed based on the reference generator outputs. Hence, three DOF would be available [16] and the yaw moment could be selected to achieve further efficiency. $M_{z}$ can be either calculated in the high level controller as a function of vehicle state [23] or it can be implicitly set by the low level control algorithm [15].

Concerning efficiency, the main vehicle power losses are caused by motor, inverter and tyres (i.e. due to longitudinal and lateral slip losses) [32]. The contributions [30, 33] propose fast parametric torque allocation strategies based on the experimental assessment of the drivetrain power losses. Specifically [33] interpolates the drivetrain power losses with a cubic polynomial function of the torque demand, whose coefficients are dependent on motor speed. The effectiveness of a cubic function is confirmed in [34] and a similar formulation is also used in [35]. In [15, 19] different efficiency optimisation functions are considered and compared, showing that optimising slip losses might be slightly better than optimising input power losses, depending on the motor technology. However, relying on tyre slips may not actually be viable as they are not directly measurable and their estimation is still challenging [36]. In [23] a rule-based approach is proposed considering losses due to motor, inverter, transmission and longitudinal slip, along with the hypothesis of 4 identical and independent motors characterised by power losses expressed as a cubic polynomial function of the torque demand.

This paper proposes a novel TV control framework for electric vehicles with multiple motors, schematised in Fig. 1. While the majority of the approaches in the literature focus only on one of blocks (a), (b), (c), the method proposed in this paper encompasses them all at the same time. This allows a smooth integration between each block, since practically the design of each block is not completely independent from each other.

The designed control framework includes the possibility for the driver to select different driving modes, to accommodate various desirable vehicle behaviours depending on the driving conditions. When vehicle handling is a priority, one of the available handling modes is selected: the reference generator is active, the high level controller interprets the driver input, and the low level controller allocates wheel torque according to the high level controller commands, at the same time minimising power consumption (solid line in Fig. 1). Instead, when the priority is energy efficiency, the reference generator is not active, and the yaw moment is defined directly in the low level controller based on an energy efficiency logic (dashed line in Fig. 1). For the first time the proposed TV control framework allows to select driving modes that prioritise either handling or energy efficiency.

The remainder of this paper is structured as follows. Section 2 describes the developed TV control framework, with a subsection per each block. Section 3 deals with the validation of the proposed strategy on a high fidelity vehicle model. Concluding remarks are in Sect. 4.

\section{Torque-vectoring control framework}

This section presents the proposed integrated solution for each of the main blocks depicted in Fig. 1. The vehicle is assumed to feature four identical electric 


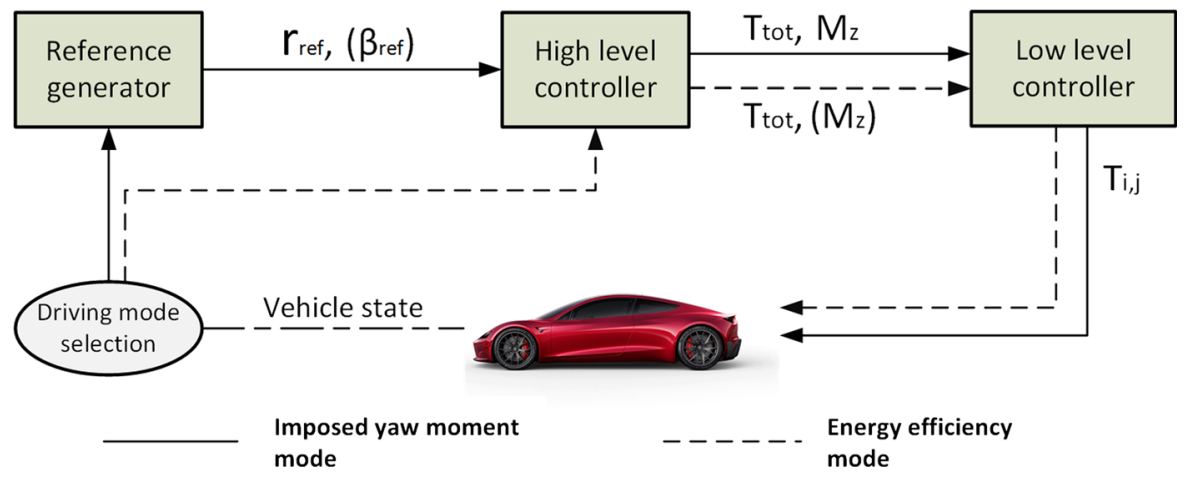

Fig. 1 Scheme of the proposed TV control framework, including the "Driving mode selection" block

motors, i.e. one per wheel, which is a typical configuration. The sensory requirements for the implementation of the proposed control framework are discussed in Sect. 2.5.

\subsection{Reference generator}

The reference generator elaborates a reference yaw rate and a reference sideslip angle. For the reference yaw rate, the proposed solution is inspired to the quasisteady-state approach proposed in [20], which entails the full design of a reference understeer characteristic, i.e. the relationship between dynamic steering wheel angle, $\delta_{d y n}$, and lateral acceleration, $a_{y}$. Compared to approaches that adopt a mere linear behaviour, this method allows to design understeer characteristics with a realistic shape. On the other hand, such design freedom might tempt the designer, who might choose potentially any curve. However not only that may produce an unnatural vehicle behaviour, but also excessive deviations from the behaviour of the baseline vehicle may require a too severe control action which might not be practically achievable by the TV controller.

Figure 2 shows a typical understeer characteristic (baseline vehicle, in black) and two potential characteristics achievable by TV. Desirable targets of the TV-based design of the understeer characteristic include: (1) extension of the linear region; (2) increase of the maximum lateral acceleration; and (3) increase of the steering responsiveness of the vehicle. That is achieved by tuning the three main parameters that define the curve, each having a clear physical meaning:
- $K_{u s}$ is the understeer gradient and it represents the initial slope of the curve. For example, a value of $K_{u s}$ lower than for the baseline vehicle results in a more reactive vehicle (closer to a neutral behaviour).

- $a_{y}^{*}$ is the lateral acceleration limit of the linear region.

- $a_{y, M A X}$ is the maximum achievable lateral acceleration.

The reference yaw rate is obtained by solving the following equation with respect to $r_{\text {ref,S }}$, using the steady-state relationship $\frac{a_{y}}{V}=r_{\text {ref }, S}[37]$ :

$$
\begin{aligned}
\delta_{s w}\left(a_{x}, a_{y}, V, \mu\right) & =\delta_{d y n}\left(a_{x}, a_{y}, \mu\right)+\delta_{k i n} \\
& =\delta_{d y n}\left(a_{x}, a_{y}, \mu\right)+\tau\left(\frac{l}{R_{c}}\right) \\
& =\delta_{d y n}\left(a_{x}, a_{y}, \mu\right)+\tau\left(l \frac{r_{r e f, S}}{V}\right)
\end{aligned}
$$

where $\delta_{s w}$ is the steering wheel angle - formed by two contributions, i.e. the dynamic steering wheel angle and the kinematic steering wheel angle $-\tau$ is the

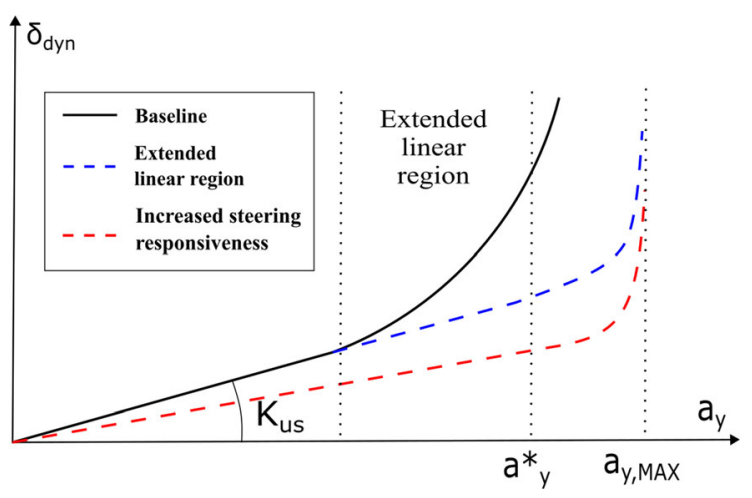

Fig. 2 Design of the vehicle understeering characteristic within the reference generator 
steering ratio, $V$ the vehicle speed, $l$ the vehicle wheelbase, $\mu$ the estimated tyre-road friction coefficient, $R_{c}$ the radius of curvature [37] $\left(R_{c}=\frac{V}{r_{\text {ref }, S}}\right.$ in steady-state conditions or in quasi-steady-state conditions with small sideslip angles), $a_{x}$ and $a_{y}$ the longitudinal and lateral accelerations, respectively.

$\delta_{d y n}$ is computed by inverting Eq. 2 [20], which is the adopted analytical formulation for the desired understeer characteristic (Fig. 2):

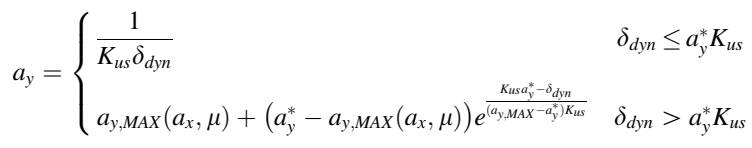

The maximum lateral acceleration, $a_{y, M A X}$, is calculated based on the estimated maximum achievable lateral force:

$a_{y, M A X}=\sum \frac{F_{y, i j}^{M A X}}{m}$

where $i=1,2$ respectively for front and rear axle, $j=$ 1,2 respectively for left and right side, $m$ is the vehicle mass, and the maximum achievable lateral force per wheel, $F_{y, i j}^{M A X}$, is calculated as:

$$
F_{y, i j}^{M A X}=\sqrt{[\underbrace{\left(d_{1}+d_{2} \frac{F_{z, i j}-F_{z, 0}}{F_{z, 0}}\right)\left(F_{z, i j}\right)}_{D=\mu F_{z, i j}=F_{t o t, i j}^{M A X}}]^{2}-\left[F_{x, i j}\right]^{2}}
$$

in which $D$ is worked out based on the Pacejka Magic Formula [38] using the coefficients $d_{1}, d_{2}$ and $F_{z, 0}$, and the wheel vertical loads, $F_{z, i j}$, are:

$$
\begin{aligned}
F_{z, i j} & =F_{z, s t a t, i j}+\Delta F_{z, i j} \\
& =F_{z, s t a t, i j}+\frac{-1^{i}}{2} \frac{m h a_{x}}{l}+\frac{-1^{j}}{2} \frac{m h a_{y}}{w} x_{i}
\end{aligned}
$$

where $F_{z, \text { stat }, i j}$ is the static load on wheel $i j, w$ is the vehicle track, and $x_{i}$ is the lateral load transfer distribution coefficient between front and rear axle $\left(x_{1}+x_{2}=1\right)$. In Eq. $4, F_{y, i j}^{M A X}$ is calculated according to the combined tyre-road interaction (longitudinal and lateral), based on the maximum force available at each tyre, $F_{t o t, i j}^{M A X}=D=\mu F_{z, i j}$, and the longitudinal force required at each tyre, approximated as $F_{x, i j}=\frac{1}{4} m a_{x}$.
Finally, the value of $a_{y, M A X}$ obtained combining Eqs. 3, 4 and 5 is saturated with the value that would zero the vertical load on any wheel (incipient vehicle rollover). The saturation value is calculated by solving Eq. 5 replacing the generic $a_{y}$ with $a_{y, M A X}$.

Based on the above, Eq. 1 can be solved offline and the solution can be stored in a look-up table, so that the reference yaw rate, $r_{r e f}$, can be obtained as a function of steering wheel angle, vehicle speed, longitudinal acceleration and a first order filter. An example is shown in Fig. 3.

The reference sideslip angle, $\beta_{\text {ref }}$, is defined as a function of the actual value, $\beta$, as follows [18]:

$\beta_{\text {ref }}=\beta_{M A X} \tanh \left(\frac{\beta}{\beta_{M A X}}\right)$

where $\beta_{M A X}$ is a constant. Essentially the sideslip angle is bounded within $\pm \beta_{M A X}$.

\subsection{High level controller}

The high level controller elaborates the desired values of total torque and yaw moment (Fig. 1). The total torque, $T_{t o t}$, is worked out through a driveability map based on vehicle velocity and driver input (accelerator/brake pedal positions). Also a simple cruise control logic was implemented, i.e. a PID controller based on the difference between reference and current value of the vehicle speed.

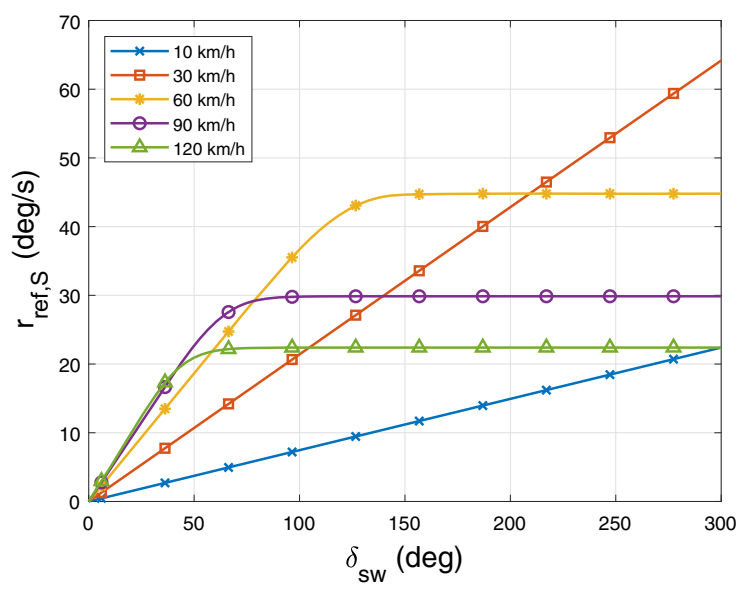

Fig. 3 Example of the reference yaw rate map as a function of the steering wheel angle for different values of vehicle speed, $a_{x}=0$ 
The calculation of the desired yaw moment, $M_{z}$, is inspired to the approach presented in [18]. Specifically:

$$
M_{z}=f\left(I_{Y}\right) M_{z, S S C}+M_{z, T C}=f\left(I_{Y}\right) M_{z, S S C}+k_{Y} I_{Y}
$$

where the first contribution is an optimal yaw moment for steady-state conditions, $M_{z, S S C}$, and the second contribution, $M_{z, T C}$, ensures stability in transient conditions [39]. $k_{Y}$ is a control gain, $I_{Y}$ is denoted as "yaw index" and it identifies whether the vehicle is in transient or steady-state conditions, $f$ is a function of $I_{Y} . f$ and $I_{Y}$ allow to weigh between the two contributions based on the vehicle conditions. For instance, when the vehicle is in steady-state conditions then $I_{Y}=0$ and $f\left(I_{Y}\right)=1$.

$I_{Y}$ is defined as:

$$
I_{Y}=\frac{a_{y}}{V}-r
$$

$I_{Y}$ is based on signals that are measured on-board by controllers such as Electronic Stability Control (ESC), which is a mandatory safety system in modern cars. An intuitive justification of Eq. 8 can be inferred by analysing the definition of lateral acceleration in case of constant speed and small sideslip angles [40]:

$$
a_{y}=V \dot{\beta}+r V
$$

which can be rearranged for $\dot{\beta}$, obtaining Eq. 8. This means that the $M_{z, T C}$ contribution prevents $\beta$ from excessively increasing/decreasing, which is an indicator of potential incipient loss of vehicle stability [41].

The function $f$ is defined as:

$$
f\left(I_{Y}\right)=\frac{1}{2}\left(1-\tanh \left(c_{1}\left|I_{Y}\right|+c_{2}\right)\right)
$$

where $c_{1}$ and $c_{2}$ are constants, to be carefully selected by the control engineer. $c_{1}$ is measured in s (note the unit of $I_{Y}$ is $\left.\mathrm{rad} / \mathrm{s}\right), c_{2}$ is unitless. In particular, recommended ranges are:

- $0<c_{1} \leq 100$ because $c_{1}>0$ guarantees a bellshaped $f$, while excessive values of $c_{1}$ are avoided as they would provoke a transition of $f$ from 0 to 1 in an too tight range of $I_{Y}$, resulting in a too fast transition from $M_{z, S S C}$ to $M_{z, T C}$ according to Eq. 7, in turn implying large oscillations of $M_{z}$ that are undesired
- $-6 \leq c_{2} \leq-3$ because $c_{2} \leq-3$ guarantees $f(0)=1$, while excessively negative values of $c_{2}$ are avoided as they enlarge the range of $I_{Y}$ for which $f \approx 1$, and a too large range would not allow $M_{z, T C}$ to intervene

These effects are analysed in Fig. 4. The selected values of $c_{1}$ and $c_{2}$ are respectively $25 \mathrm{~s}$ and -3 .

$M_{z, S S C}$ is based on a Linear Quadratic Regulator (LQR). The reference vehicle model is the single track model [40]:

$$
\left\{\begin{array}{c}
m V(\dot{\beta}+r)=Y_{\beta} \beta+Y_{r} r+C_{1} \delta \\
J_{z} \dot{r}=N_{\beta} \beta+N_{r} r+a C_{1} \delta+M_{z}
\end{array}\right.
$$

where $J_{z}$ if the vehicle mass moment of inertia with respect to a vertical axis, the stability derivatives $Y_{\beta}$, $Y_{r}, N_{\beta}$ and $N_{r}$ are defined as:

$$
\begin{aligned}
Y_{\beta} & =-\left(C_{1}+C_{2}\right) \\
Y_{r} & =-\frac{\left(a C_{1}-b C_{2}\right)}{V} \\
N_{\beta} & =-\left(a C_{1}-b C_{2}\right) \\
N_{r} & =-\frac{\left(a^{2} C_{1}+b^{2} C_{2}\right)}{V}
\end{aligned}
$$

and $C_{1}$ and $C_{2}$ are, respectively, the cornering stiffness of the front and rear axle.

The system dynamics in Eq. 11 can be rearranged and written in a compact state-space representation as:

$\dot{x}=[A] x+\left[B_{M_{z}}\right] M_{z}+\left[B_{\delta}\right] \delta$

with the state vector being

$$
x=\left[\begin{array}{l}
\beta \\
r
\end{array}\right]
$$

and $A, B_{M_{z}}, B_{\delta}$ denoting respectively the dynamic matrix, the input matrix and the disturbance matrix:

$$
\begin{aligned}
& A=\left[\begin{array}{ll}
\frac{Y_{\beta}}{m V} & \frac{Y_{r}}{m V}-1 \\
\frac{N_{\beta}}{J_{z}} & \frac{N_{r}}{J_{z}}
\end{array}\right] \\
& B_{M_{z}}=\left[\begin{array}{c}
0 \\
\frac{1}{M_{z}}
\end{array}\right]
\end{aligned}
$$




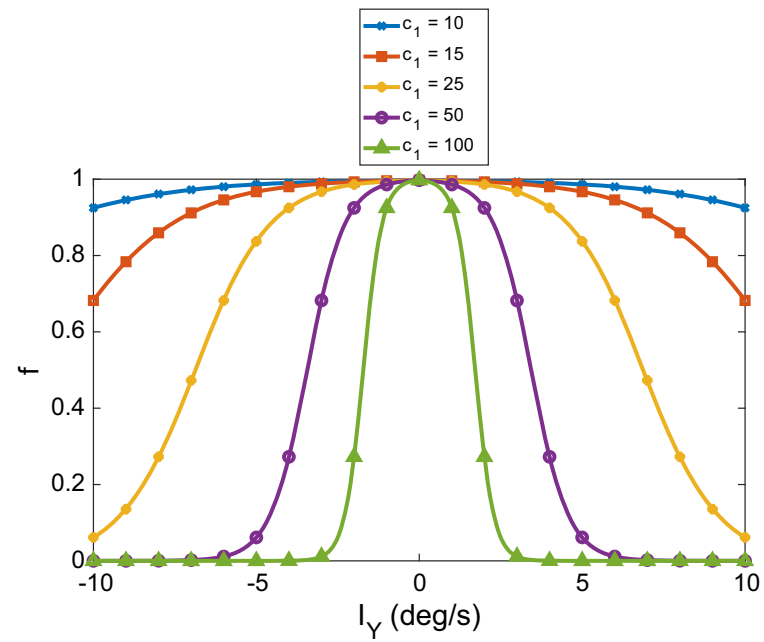

(a)

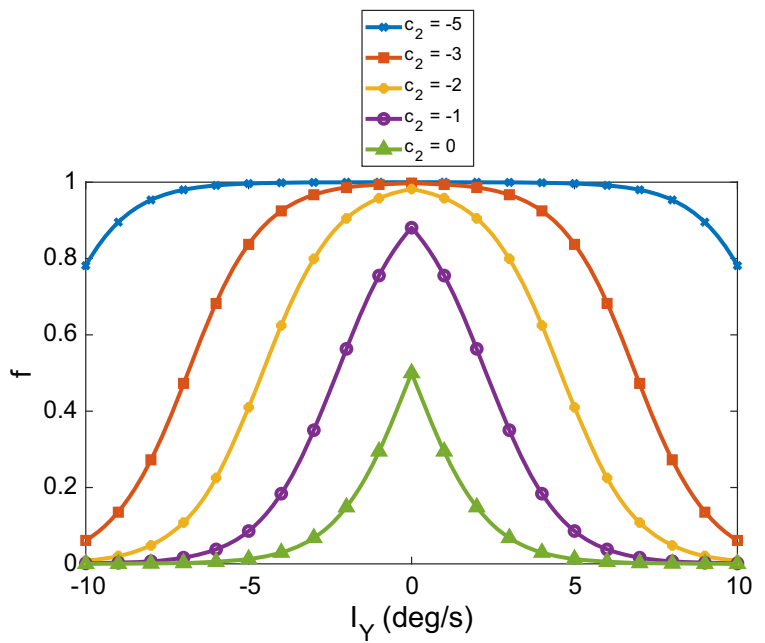

(b)

Fig. 4 Sensitivity analysis of $f\left(I_{Y}\right)$ with respect to: $\mathbf{a} c_{1}$, for $c_{2}=-3$; $\mathbf{b} c_{2}$, for $c_{1}=25 \mathrm{~s}$

$$
B_{\delta}=\left[\begin{array}{c}
\frac{C_{1}}{m V} \\
\frac{C_{1} a_{1}}{J_{z}}
\end{array}\right]
$$

Note that the wheel steer angle, $\delta$, is formally assumed as a disturbance because it cannot be controlled.

The control input $M_{z, S S C}$ is calculated by minimising the following performance index:

$$
J=\int_{0}^{\infty}\left(e^{T}[Q] e+M_{z, S S C}[R] M_{z, S S C}\right) d t
$$

where the error, $e$, is defined as:

$$
e=\left[\begin{array}{c}
\beta_{r e f}-\beta \\
r_{r e f}-r
\end{array}\right]
$$

and the symmetric and positive definite weight matrices $Q$ and $R$ are chosen as indicated in [42]:

$$
Q=\left[\begin{array}{cc}
\frac{1}{\beta_{M A X}^{2}} & 0 \\
0 & \frac{1}{r_{M A X}^{2}}
\end{array}\right] \quad R=\frac{1}{M_{z, M A X}}
$$

where $r_{M A X}=0.85 \frac{\mu g}{V}$ [43], $g$ is the gravitational acceleration, and $M_{z, M A X}$ is the maximum achievable yaw moment, which mainly depends on the electric motor characteristics. $Q$ and $R$ penalise excessive error and excessive control action, respectively, with the aim of bringing the state error as close as possible to 0 but bearing in mind that the necessary control action needs to be feasible [44]. By solving a suitable Riccati equation, a matrix $G$ is obtained so that $M_{z, S S C}=G e$. Because the single track model assumes constant vehicle speed, a gain scheduling approach is implemented, with six values of speed, i.e. $40 \mathrm{~km} / \mathrm{h}, 60 \mathrm{~km} /$ $\mathrm{h}, 80 \mathrm{~km} / \mathrm{h}, 100 \mathrm{~km} / \mathrm{h}, 120 \mathrm{~km} / \mathrm{h}$ and $140 \mathrm{~km} / \mathrm{h}$.

\subsection{Low level controller}

The low level controller elaborates the four wheel torque demands, $T_{i j}$. Under the fair assumption that the front and rear track widths are the same [33], $T_{i j}$ can be written in the general form:

$$
\left\{\begin{array}{c}
T_{11}=\left(\frac{T_{t o t}}{2}+\Delta T_{L R}\right) \sigma_{L} \\
T_{21}=\left(\frac{T_{t o t}}{2}+\Delta T_{L R}\right)\left(1-\sigma_{L}\right) \\
T_{12}=\left(\frac{T_{t o t}}{2}-\Delta T_{L R}\right) \sigma_{R} \\
T_{22}=\left(\frac{T_{t o t}}{2}-\Delta T_{L R}\right)\left(1-\sigma_{R}\right)
\end{array}\right.
$$

Equation 23 include four parameters (corresponding to four DOF):

1. The total torque, $T_{t o t}$, always imposed by the high level controller

2. The torque unbalance between left and right side, $\Delta T_{L R}$ 
3. The front-to-total wheel torque distribution factor for the left vehicle side, $\sigma_{L}=\frac{T_{11}}{T_{11}+T_{21}}$

4. The front-to-total wheel torque distribution factor for the right vehicle side, $\sigma_{R}=\frac{T_{12}}{T_{12}+T_{22}}$

$\Delta T_{L R}$ can be calculated based on the desired yaw moment, $M_{z}$, as:

$$
\Delta T_{L R}=\frac{M_{z} R_{w}}{w}
$$

where $R_{w}$ is the average wheel radius.

Once $\Delta T_{L R}$ is known, the overall torque demands at the left and right vehicle sides, respectively $T_{L}$ and $T_{R}$, are known:

$$
\begin{aligned}
& T_{L}=T_{11}+T_{21}=\frac{T_{t o t}}{2}+\Delta T_{L R} \\
& T_{R}=T_{12}+T_{22}=\frac{T_{t o t}}{2}-\Delta T_{L R}
\end{aligned}
$$

As a result, the optimisation problem reduces to two independent sub-problems, one per side. Hence, the discussion hereinafter will refer to the determination of the optimal front-to-total wheel torque distribution factor, $\sigma$, for a generic vehicle side. This approach is then used to calculate both $\sigma_{L}$ and $\sigma_{R}$.

A very relevant parameter is the second derivative (i.e. the concavity) of the drivetrain power loss as a function of the torque demand. According to [30, 33], in case the concavity is always positive, the optimal solution is the even distribution of the torque between front and rear motor $(\sigma=0.5)$. On the other hand, if the concavity is negative then the ideal solution is to distribute the torque on one wheel only $(\sigma=0$ or $\sigma=1$ ). Clearly, in general the concavity might vary, thus the optimal value of $\sigma$ depends on the amount of torque required and on the shape of the curve.

Here, the Hybrid Control Allocation (H-CA) approach proposed in [33] is adopted. For drivetrain power losses that can be approximated as third order polynomial functions of the torque demand, a switching torque $T_{s w}(V)$ is defined for each vehicle speed $V$ as the side torque demand for which the solutions $\sigma=0, \sigma=0.5$ and $\sigma=1$ are optimal and equivalent. $T_{s w}(V)$ can be calculated from the drivetrain power losses. Given the vehicle speed, the optimal solution is $\sigma=1$ (or, equivalently, $\sigma=0$, however the former is preferred for vehicle safety reasons) if the side torque demand is lower than $T_{s w}(V)$, and $\sigma=0.5$ when the side torque demand is greater than $T_{s w}(V)$.
Summing up, the algorithm used at each time step is:

$$
\begin{array}{rlrl}
\text { if } & T_{L} & >T_{s w}(V) \\
& \sigma_{L} & =0.5 \\
\text { else } & & \sigma_{L} & =1 \\
\text { if } & & T_{R} & >T_{s w}(V) \\
& \sigma_{R} & =0.5 \\
\text { else } & \sigma_{R} & =1
\end{array}
$$

Finally, it should be mentioned that the above strategy is appropriately overruled in case of wheel torque saturation, to ensure that the motor torque demands are always feasible.

\subsection{Driving modes}

A number of driving modes are introduced in the TV framework, each corresponding to a different vehicle behaviour, to accommodate different needs due to specific driving conditions and/or driver needs or preferences. Table 1 shows the key features of each driving mode, including the chosen values of $K_{u s}$ and $\beta_{M A X} . K_{u s, b}$ is the understeer gradient of the baseline vehicle, obtained with the high fidelity model described in Sect. 3.

In Normal mode and Sport mode the yaw moment is defined by the high level controller based on handling requirements (reference tracking), as per Sect. 2.1. While the Normal mode replicates the understeer gradient of the baseline vehicle, the Sport mode achieves a reduced understeer gradient hence an increased steering responsiveness. Then, the remaining two DOF $\left(\sigma_{L}\right.$ and $\left.\sigma_{R}\right)$ are chosen based on energy efficiency criteria, as discussed in Sect. 2.3.

For the first time a driving mode denoted as "Energy efficiency" mode is introduced, in which no handling requirements are imposed. When the Energy efficiency driving mode is selected, the reference generator is bypassed and $M_{z}$ is indirectly calculated within the low level controller, that manages the available three DOF $\Delta T_{L R}, \sigma_{L}$ and $\sigma_{R}$ with the only target of minimising energy consumption. This is stressed in the third and fourth columns of Table 1, in particular the fourth column indicates the torque distribution parameters defined in the low level controller for each driving mode, i.e. three parameters for the Energy efficiency mode and two parameters for the other modes. 
Table 1 Design parameters for each driving mode

\begin{tabular}{lllll}
\hline Driving mode & $K_{u s}$ & $\beta_{M A X}\left(^{\circ}\right)$ & Yaw moment definition & Torque distribution parameters \\
\hline Normal & $K_{u s, b}$ & 5 & Reference tracking & $\sigma_{L}, \sigma_{R}$ \\
Sport & $\frac{3}{4} K_{u s, b}$ & 5 & Reference tracking & $\sigma_{L}, \sigma_{R}$ \\
Energy efficiency & - & - & Energy optimisation & $\Delta T_{L R}, \sigma_{L}, \sigma_{R}$ \\
\hline
\end{tabular}

\subsubsection{The energy efficiency mode}

The Energy efficiency mode aims at minimising the overall vehicle power losses, which are mainly due to the motor-inverter losses and tyre slip losses [32]. So, $\Delta T_{L R}$ needs to be calculated in order to minimise power losses, and not based on reference tracking.

Assuming that the drivetrain power losses are a cubic polynomial function of the torque demand, [23] shows that for low-medium values of longitudinal force (normal driving conditions for a passenger car), the drivetrain power losses are minimised by a yaw moment that allocates the whole torque demand on either side of the vehicle. And, to minimise tyre slip power losses at the same time, the overall torque demand should be on the external side of the vehicle. ${ }^{1}$

Based on the above, according to Eq. 23, it should be $\Delta T_{L R}=\operatorname{sign}\left(\delta_{s w}\right) \frac{T_{t o t}}{2}$. Note that the adopted sign convention implies positive steering angle, yaw rate and yaw moment when clockwise. To avoid unnatural vehicle behaviour, a deadband is applied for relatively small values of steering wheel angle, with a threshold $\delta_{s w, t h}= \pm 20^{\circ}$. The motor torque limits are also accounted for, i.e., if $T_{t o t}$ exceeds the nominal torque available on the vehicle side, then the excess torque is assigned to the inner side of the vehicle.

Summing up, for the Energy efficiency mode $\Delta T_{L R}$ is selected as follows:

\footnotetext{
${ }^{1}$ It is worth to note that, hypothetically, assigning more than the overall torque demand on the external vehicle side would imply a negative (regenerative) torque on the inner side, which is far from optimal [33].
}

$$
\begin{aligned}
\text { if } \quad T_{\text {max }, \text { side }} \geq T_{\text {tot }} \text { AND }\left|\delta_{s w}\right|>\delta_{s w, t h} \\
\Delta T_{L R}=\operatorname{sign}\left(\delta_{s w}\right) \frac{T_{t o t}}{2} \\
\text { else if } \quad\left|\delta_{s w}\right|>\delta_{s w, t h} \\
\Delta T_{L R}=\operatorname{sign}\left(\delta_{s w}\right)\left(T_{\text {max }, \text { side }}-\frac{T_{\text {tot }}}{2}\right) \\
\text { else } \quad \Delta T_{L R}=0
\end{aligned}
$$

where the maximum torque $T_{\text {max }}$,side is computed based on the motor characteristic (e.g. Fig. 5), i.e. the curve providing the maximum torque for motor $i j, \Gamma_{\max , i j}$, as a function of the motor angular speed, $\Omega_{i j}$ (again, $i=$ 1,2 respectively for front and rear axle, $j=1,2$ respectively for left and right side):

$$
T_{\max , \text { side }}=\tau\left(\Gamma_{\max , 1 q}\left(\Omega_{1 q}\right)+\Gamma_{\max , 2 q}\left(\Omega_{2 q}\right)\right)
$$

in which $\tau$ is the transmission ratio, and the subscript $q$ refers to the external side of the vehicle, which can be automated using the expression ${ }^{2}$ $q=\left(\frac{1}{\sqrt{2}}^{\operatorname{sign}\left(\delta_{s w}\right)-1}\right)$

It should be noted that the driving style has a large impact on power consumption, too: energy efficiency optimisation algorithms cannot be much effective if associated with an aggressive driving style. It is therefore assumed that the driving style is appropriate according to the selected driving mode.

\subsection{Measurements and sensors}

The proposed controller is designed to exploit only sensors which are nowadays available on all passenger cars and which are used by other active control systems, such as the Anti-lock Braking Systems (ABS)

\footnotetext{
${ }^{2}$ When the steering wheel angle, $\delta_{s w}$, is positive, the vehicle is turning right and $\left(\frac{1}{\sqrt{2}}^{\operatorname{sign}\left(\delta_{s w}\right)-1}\right)=1$, so the left side (1) is the external side; when $\delta_{s w}$, is negative, the vehicle is turning left and $\left(\frac{1}{\sqrt{2}} \operatorname{sign}\left(\delta_{s w}\right)-1\right)=2$, so the right side (2) is the external side.
} 


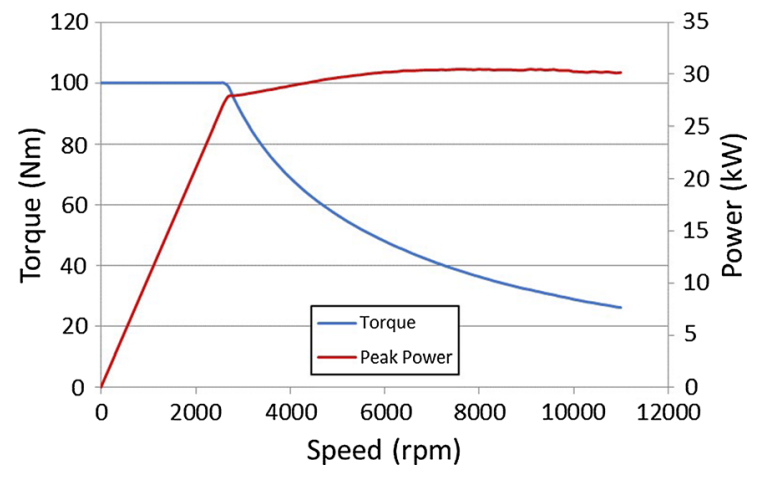

Fig. 5 Motor characteristic curves: torque and power as functions of the angular speed

and the Electronic Stability Control (ESC). Table 2 reports the required measurements and the corresponding sensors. These measurements are available on the vehicle Controlled Area Network (CAN) bus.

Typical technical data of standard automotive sensors are reported in Table 3 (steering wheel angle sensor [45]) and Table 4 (IMU [46, 47]).

Based on the measurements reported in Table 2, vehicle speed $V$ and sideslip angle $\beta$ are estimated by the control algorithm. Specifically, the vehicle speed is estimated based on the measurements of wheel speeds (with frequency ranging from 0 to $20 \mathrm{kHz}$ [48]) and longitudinal acceleration. Consolidated fuzzy estimation algorithms are used on this purpose by ABS control systems $[49,50]$. The vehicle sideslip angle is estimated through algorithms such as in [51, 52], again based on standard automotive sensors like those reported in Tables 2, 3 and 4.

\section{Results and discussion}

The effectiveness of the proposed TV control strategy was assessed by means of numerical simulations. Both steady-state and transient manoeuvres were performed, to evaluate the ability of the controller to improve different aspects of the vehicle dynamics. Simulations were carried out on a 14 DOF vehicle model, developed for MATLAB-Simulink and validated against experimental tests performed on an instrumented passenger car (segment D) [53]. The DOF of the model are:

- 6 DOF for the car body, i.e. 3 displacements and 3 rotations (yaw, pitch and roll).

- 4 DOF for the wheel vertical displacements.

- 4 DOF for the wheel rotations with respect to their hub axis.

The tyre-road interaction forces are modelled through a Pacejka Magic Formula [38], including combined slip effect and relaxation lengths. The main vehicle parameters are reported in Table 5.

The vehicle features four identical on-board electric motors. Their torque and power characteristics are depicted in Fig. 5. The trasmission gear ratio is indicated in Table 5. To account for the electric motor torque regulator dynamics, a first order time lag was introduced in the model $[3,18]$. The power losses of each motor, $P_{l o s s, i j}$, are defined as functions of motor torque, $\Gamma_{i j}$, and motor speed, $\Omega_{i j}$, as the difference between the input power, $P_{i n}$, and the output power, $P_{\text {out }}$ :

Table 3 Steering wheel angle sensor technical data

\begin{tabular}{llll}
\hline Range & Angular speed & Resolution & CAN Speed \\
\hline $\pm 780^{\circ}$ & up to $1000^{\circ} / \mathrm{s}$ & $0.1^{\circ}$ & $500 \mathrm{kbaud}$ \\
\hline
\end{tabular}

Table 2 Required measurements for the proposed control system, and corresponding sensors already available on modern passenger cars

\begin{tabular}{lll}
\hline Measurement & Symbol(s) & Sensor \\
\hline Yaw rate & $r$ & Inertial Measurement Unit (IMU) \\
Longitudinal and lateral accelerations & $a_{x}, a_{y}$ & Inertial Measurement Unit (IMU) \\
Wheel speed & $\omega_{i j}$ & Wheel speed sensors \\
Steering wheel angle & $\delta_{s w}$ & Steering wheel angle sensor (LWS) \\
\hline
\end{tabular}


Table 4 IMU technical data

\begin{tabular}{lllll}
\hline & Range & Filter & Resolution & CAN Speed \\
\hline Gyroscope & $\pm 300 \% \mathrm{~s}$ & $70 \mathrm{~Hz}$ (configurable) & $0.1 \% \mathrm{~s}$ & $500 \mathrm{kbaud}$ \\
Accelerometer & $\pm 50 \mathrm{~m} / \mathrm{s}^{2}$ & $70 \mathrm{~Hz}$ (configurable) & $0.1 \mathrm{~m} / \mathrm{s}^{2}$ & $500 \mathrm{kbaud}$ \\
\hline
\end{tabular}

Table 5 Main vehicle parameters

\begin{tabular}{lll}
\hline Symbol & Name and unit & Value \\
\hline$m$ & Mass (kg) & 1580 \\
$J_{z}$ & Moment of inertia, vertical axis $\left(\mathrm{kg} \mathrm{m}^{2}\right)$ & 2210 \\
$a$ & Front semi-wheelbase (m) & 0.977 \\
$l$ & Wheelbase (m) & 2.7 \\
$\tau$ & Motor transmission ratio (-) & 8.92 \\
$R_{w}$ & Wheel radius (m) & 0.336 \\
$w$ & Track width (m) & 1.592 \\
$h$ & Centre of mass height (m) & 0.55 \\
$C_{1}$ & Front axle cornering stiffness $(\mathrm{N} / \mathrm{rad})$ & $2.355 \times 10^{5}$ \\
$C_{2}$ & Rear axle cornering stiffness $(\mathrm{N} / \mathrm{rad})$ & $2.196 \times 10^{5}$ \\
\hline
\end{tabular}

$$
\begin{aligned}
P_{\text {loss }, i j} & =P_{\text {in }}-P_{\text {out }}=\frac{\Gamma_{i j} \Omega_{i j}}{\eta_{i j}\left(\Gamma_{i j}, \Omega_{i j}\right)}-\Gamma_{i j} \Omega_{i j} \\
& =\Gamma_{i j} \Omega_{i j} \frac{1-\eta_{i j}\left(\Gamma_{i j}, \Omega_{i j}\right)}{\eta_{i j}\left(\Gamma_{i j}, \Omega_{i j}\right)}
\end{aligned}
$$

where the efficiency $\eta_{i j}\left(\Gamma_{i j}, \Omega_{i j}\right)$ is obtained from the map shown in Fig. 6, that accounts for motor and inverter losses. Such map was interpolated with the third order polynomial proposed in [35]:

$$
\begin{aligned}
& P_{l o s s, i j}\left(\Gamma_{i j}, \Omega_{i j}\right)=\sum k_{n p}\left(\frac{\Gamma_{i j}}{\Gamma_{b}}\right)^{n}\left(\frac{\Omega_{i j}}{\Omega_{b}}\right)^{p} P_{b} \\
& n, p=1,2,3
\end{aligned}
$$

For this case study, the normalising factors $\Gamma_{b}, \Omega_{b}$ and $P_{b}$ are respectively $100 \mathrm{Nm}, 11,000 \mathrm{rpm}$ and $13 \mathrm{~kW}$. The longitudinal and lateral tyre slip power losses are calculated with the formulations in [23]. In particular, for the longitudinal tyre slip power losses, $P_{l o s s, x, i j}$ :

$$
P_{l o s s, x, i j}=F_{x, i j} v_{s l i p, x, i j}
$$

where $F_{x, i j}$ is the longitudinal force and $v_{s l i p, x, i j}$ is the longitudinal slip speed at wheel $i j$. For the lateral tyre slip power losses:

$$
P_{l o s s, y, i j}=F_{y, i j} v_{s l i p, y, i j}
$$

where $F_{y, i j}$ is the lateral force and $v_{s l i p, y, i j}$ is the lateral slip speed at wheel $i j$. Forces and slip speeds are available within the vehicle model described above.

\subsection{Steady-state manoeuvre: ramp steer}

The steady-state performance of the vehicle was evaluated through a quasi-steady-state manoeuvre, similar to [54]. The car performed a quasi-steady-state ramp steer at $60 \mathrm{~km} / \mathrm{h}$ with steering wheel angle from 0 to $60^{\circ}$ in $20 \mathrm{~s}$. The manoeuvre was executed multiple times, i.e. with the baseline vehicle and all the driving modes presented in Table 1 .

Figure 7 shows the resulting understeer characteristics, showing the extended linear region and the increased maximum lateral acceleration achieved with the TV-controlled vehicle - up to $8.92 \mathrm{~m} / \mathrm{s}^{2}$ - with respect to the baseline vehicle $-8.06 \mathrm{~m} / \mathrm{s}^{2}$. When using the Normal mode, the understeering gradient is the same as the baseline vehicle. When using the Sport mode the controller is also able to decrease the slope of the curve, resulting in increased responsiveness of the vehicle. This confirms the effectiveness of the reference generator and of the high level controller. In Energy efficiency mode the controller does not impose a specific understeer characteristic, yet there is a benefit in that the maximum lateral acceleration 


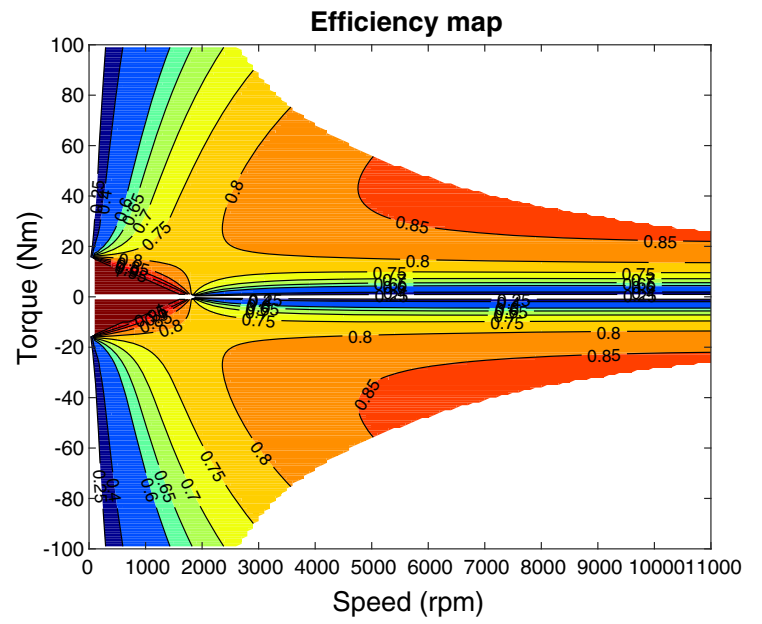

Fig. 6 Motor efficiency map showing iso-efficiency curves, obtained from the motor manufacturer

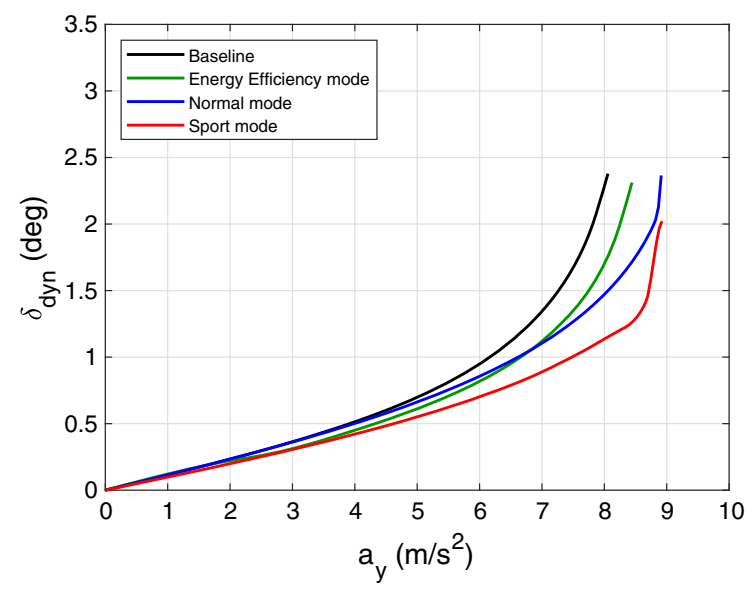

Fig. 7 Understeer characteristic of the vehicle obtained through a quasi-steady-state test

increases to $8.46 \mathrm{~m} / \mathrm{s}^{2}$. That occours since the optimal yaw moment is always concordant with the steering angle and the yaw rate. Figure 8 illustrates the power losses during the same manoeuvre, showing the effectiveness of the Energy efficiency mode that provides the minimum energy consumption for any $a_{y}$, saving up to $7.5 \%$ with respect to the baseline vehicle. On the other hand, the Sport mode provokes an increase in energy consumption. Table 6 reports the power loss variation with respect to the baseline vehicle for three levels of lateral acceleration, $\Delta P_{\text {loss,tot }}$. Interestingly, the Sport mode shows a nonmonotonic trend $\left(+1.58 \%\right.$ at $a_{y} \approx 2.5 \mathrm{~m} / \mathrm{s}^{2},+7.21$ $\%$ at $a_{y} \approx 5.4 \mathrm{~m} / \mathrm{s}^{2},+0.69 \%$ at $a_{y} \approx 8.2 \mathrm{~m} / \mathrm{s}^{2}$ ), due to the power losses being rather similar at low $a_{y}$ and to the baseline and Sport mode curves intersecting at $a_{y} \approx 8.2 \mathrm{~m} / \mathrm{s}^{2}$ in Fig. 8. The Energy efficiency mode, instead, presents increasing benefits as the lateral acceleration increases.

\subsection{Transient manoeuvres}

\subsubsection{Open loop: step steer}

An important advantage of a TV control system is the improvement of the vehicle dynamics even in transient conditions, as shown e.g. in [55]. To verify this aspect, a step steer manoeuvre was performed at $100 \mathrm{~km} / \mathrm{h}$ (the speed was maintained through a cruise control logic), with the steering wheel angle sharply increased from $0^{\circ}$ to $40^{\circ}$ and kept for $3 \mathrm{~s}$, before returning to $0^{\circ}$.

Results are reported in Fig. 9. The most significant effect of TV can be seen on the yaw rate, where the controller achieves $17 \%$ in Normal mode and $18.05 \%$ in Sport mode, with a significant decrease of overshoot and oscillations with respect to the baseline vehicle. As a consequence, also the achieved lateral acceleration is larger than for the baseline vehicle, respectively $8.2 \mathrm{~m} / \mathrm{s}^{2}$ and $8.8 \mathrm{~m} / \mathrm{s}^{2}$ for Normal mode and Sport mode. The peculiar behaviour of the yaw rate in Sport mode right after the step steer is due to the values assumed by $\beta$, and the LQR action that concurrently controls yaw rate and sideslip angle. The Energy efficiency mode shows again an increased yaw rate with respect to the baseline vehicle, but since this mode does not include a yaw rate control, there is no significant improvement in the transient behaviour.

\subsubsection{Closed loop: double lane change}

The behaviour of the vehicle in a more realistic scenario is explored through a double lane change manoeuvre. The vehicle begins the manoeuvre at 100 $\mathrm{km} / \mathrm{h}$, then the accelerator pedal is released for the remainder of the manoeuvre, in line with the recommendation of the ISO standardised test. A driver model was implemented to follow the required trajectory, as suggested in [18].

Results are reported in Fig. 10, showing that the vehicle is always able to follow the predetermined path. With respect to the baseline vehicle, the TV control helps to significantly reduce the required 


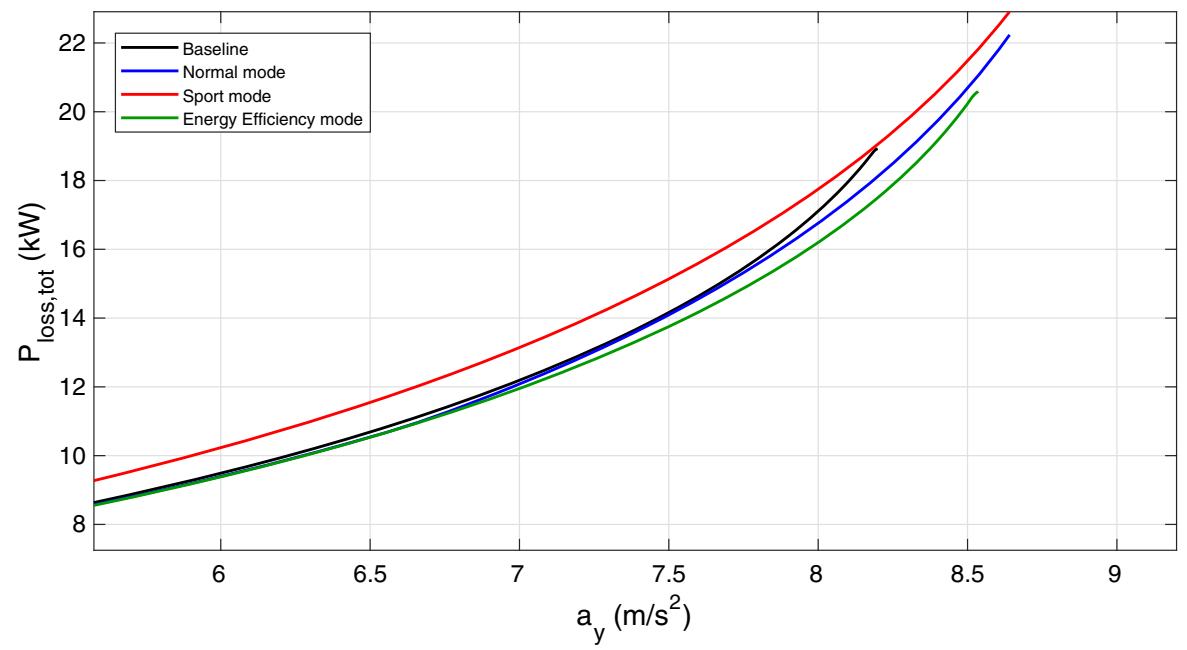

Fig. 8 Vehicle power losses as a function of the lateral acceleration obtained through a quasi-steady-state test: comparison between baseline vehicle and Normal mode, Sport mode, Energy efficiency mode

Table 6 Power loss and energy consumption variation with respect to the Baseline vehicle, for different driving modes along selected manoeuvres

\begin{tabular}{lllll}
\hline Driving mode & \multicolumn{2}{l}{ Ramp steer } & \multirow{2}{*}{\begin{tabular}{c} 
Mild slalom \\
\cline { 2 - 4 }
\end{tabular}} & \multicolumn{2}{c}{$\Delta E_{\text {loss, }, \text { ot }}(\%)$} \\
\cline { 2 - 4 } & Low $a_{y}$ & Medium $a_{y}$ & High $a_{y}$ & \\
\hline Normal mode & $-0.08 \%$ & $-0.60 \%$ & $-4.28 \%$ & $+2.82 \%$ \\
Sport mode & $+1.58 \%$ & $+7.21 \%$ & $+0.69 \%$ & $+7.01 \%$ \\
Energy efficiency mode & $-0.23 \%$ & $-0.84 \%$ & $-7.51 \%$ & $-1.40 \%$ \\
\hline
\end{tabular}

steering input by the driver during the most critical parts of the manoeuvre, whilst keeping the sideslip angle within safe limits. The yaw rate is also much smoother in the final part of the manoeuvre. The Energy efficiency mode was not tested in this configuration because the total torque demand is zero due to the accelerator pedal position, hence such mode would yield the same results of the baseline vehicle.

\subsubsection{Closed loop: mild slalom}

To further verify the effectiveness of the Energy efficiency mode, a "mild slalom" manoeuvre was simulated with a milder profile than for the double lane change, as shown in Fig. 11, and a constant total torque demand of $50 \mathrm{Nm}$. Although not representative of driving conditions on ordinary roads, the limited accelerations of the manoeuvre - far from the friction limits - are typical of standard driving conditions, where it would make sense for the driver to select the Energy efficiency mode.
The initial speed is set to $70 \mathrm{~km} / \mathrm{h}$ and the test is held in high friction conditions, again for the baseline vehicle and all the driving modes presented in Table 1.

As shown in Fig. 11, the intrinsic mild nature of the manoeuvre yields very similar trends for steering wheel angle, yaw rate, sideslip angle and lateral acceleration. More interestingly, the bottom plot of Fig. 11 shows the integral of the overall power losses, i.e. the overall energy lost, $E_{\text {loss,tot }}$. Table 6 reports the energy loss variation with respect to the baseline vehicle, $\Delta E_{\text {loss,tot }}$. The Energy efficiency mode yields on overall energy saving of $1.40 \%$ with respect to the baseline vehicle, which is deemed significant due to the mildness of the manoeuvre, and is the only case showing a benefit in terms of energy consumption. In fact, despite the Normal mode proved more efficient than the baseline vehicle for the ramp steer manoeuvre, that was not the case for the mild slalom. Specifically, Normal and Sport mode result in increased consumptions, respectively $2.82 \%$ more and $7.01 \%$ more than the baseline vehicle. Finally, it 
Fig. 9 Step steer manoeuvre on a high friction road: time histories of steering wheel angle $\left(\delta_{s w}\right)$, yaw rate $(r)$, sideslip angle $(\beta)$, lateral acceleration $\left(a_{y}\right)$
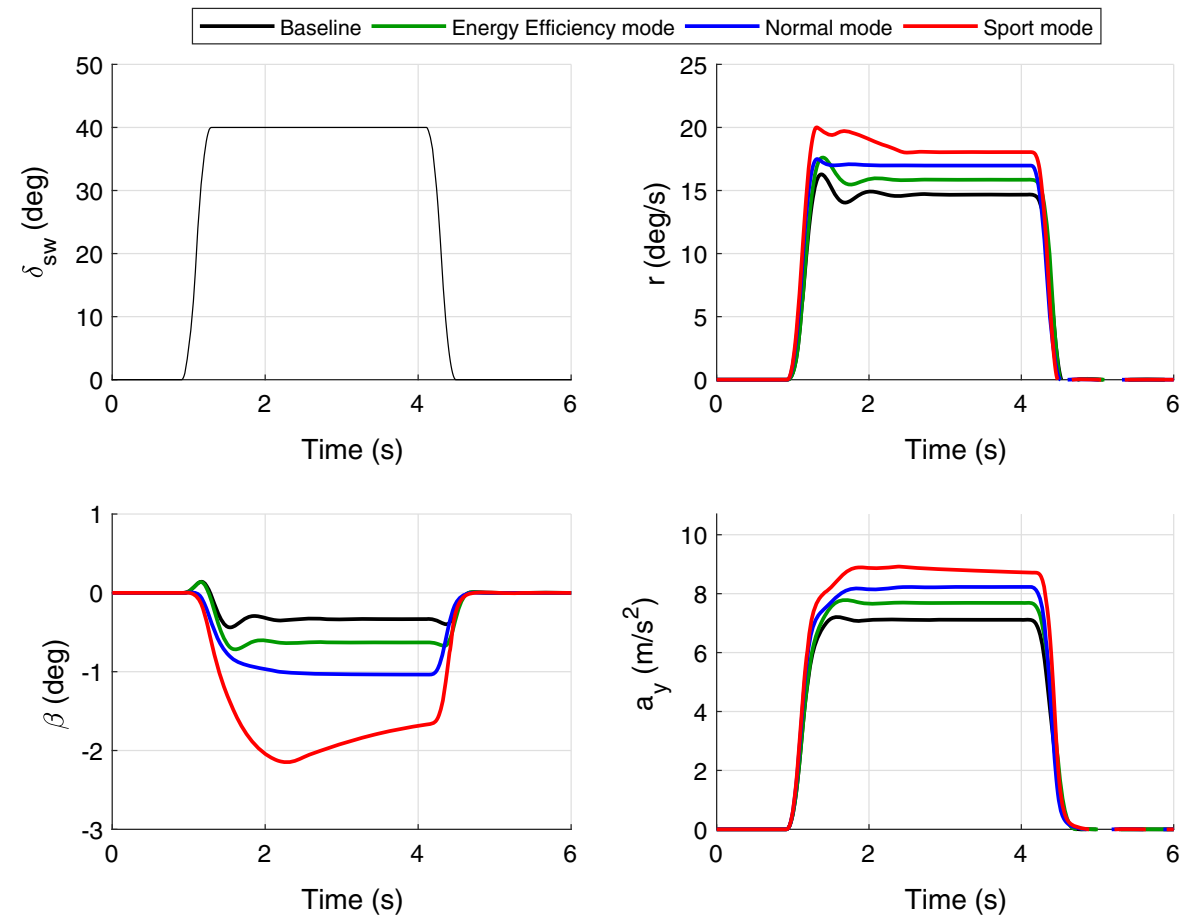

Fig. 10 Double lane change manoeuvre on a high friction road: (top) time histories of steering wheel angle $\left(\delta_{s w}\right)$, yaw rate $(r)$, sideslip angle $(\beta)$, lateral acceleration $\left(a_{y}\right)$; (bottom) reference and actual trajectory

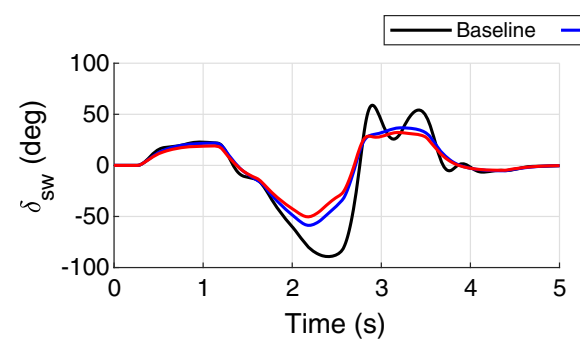

Normal mode — Sport mode
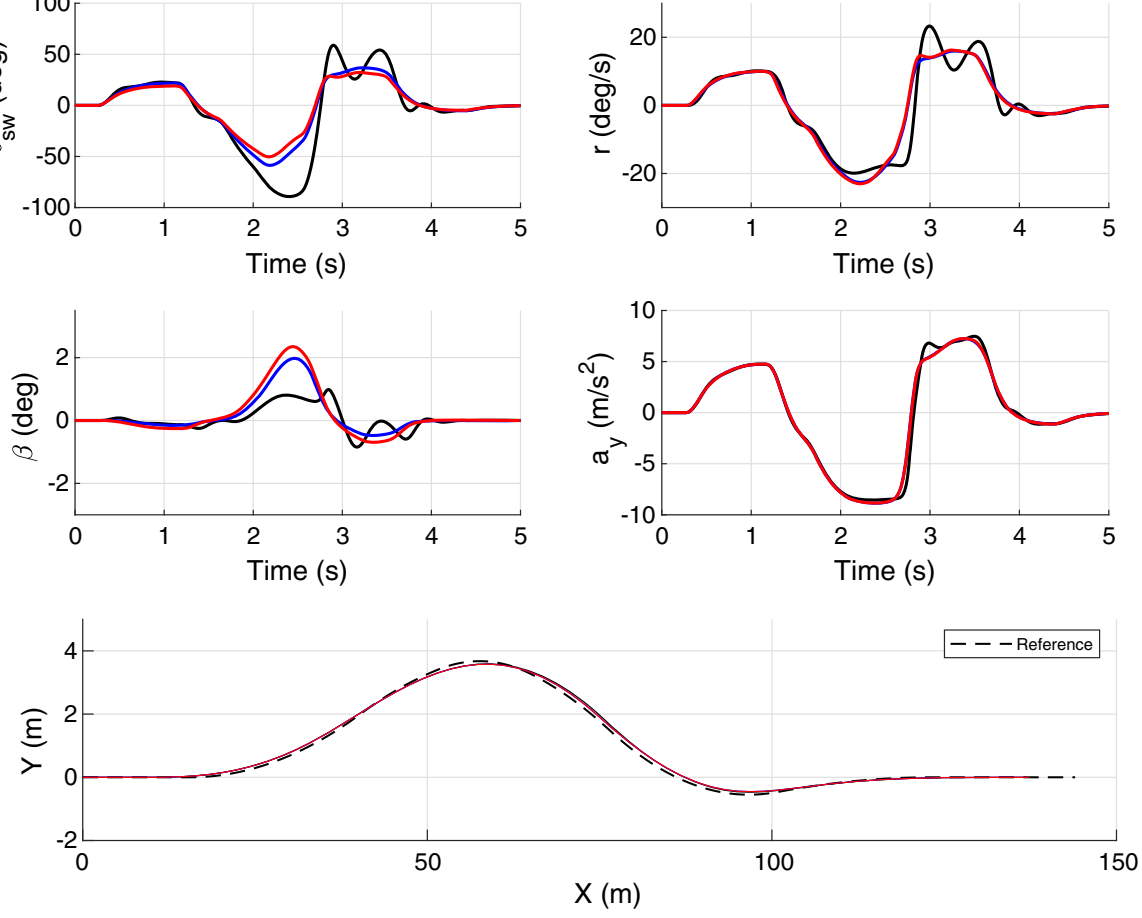
Fig. 11 Mild slalom manoeuvre on a high friction road: (top) time histories of steering wheel angle $\left(\delta_{s w}\right)$, yaw rate $(r)$, sideslip angle $(\beta)$, lateral acceleration $\left(a_{y}\right)$; (bottom) reference and actual trajectory, time history of the total energy losses $\left(E_{\text {loss }, \text { tot }}\right)$

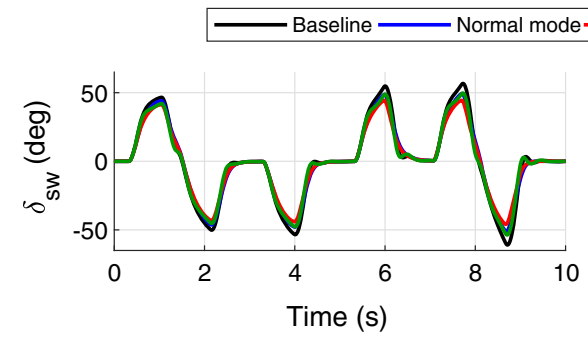

Sport mode — Energy Efficiency mode
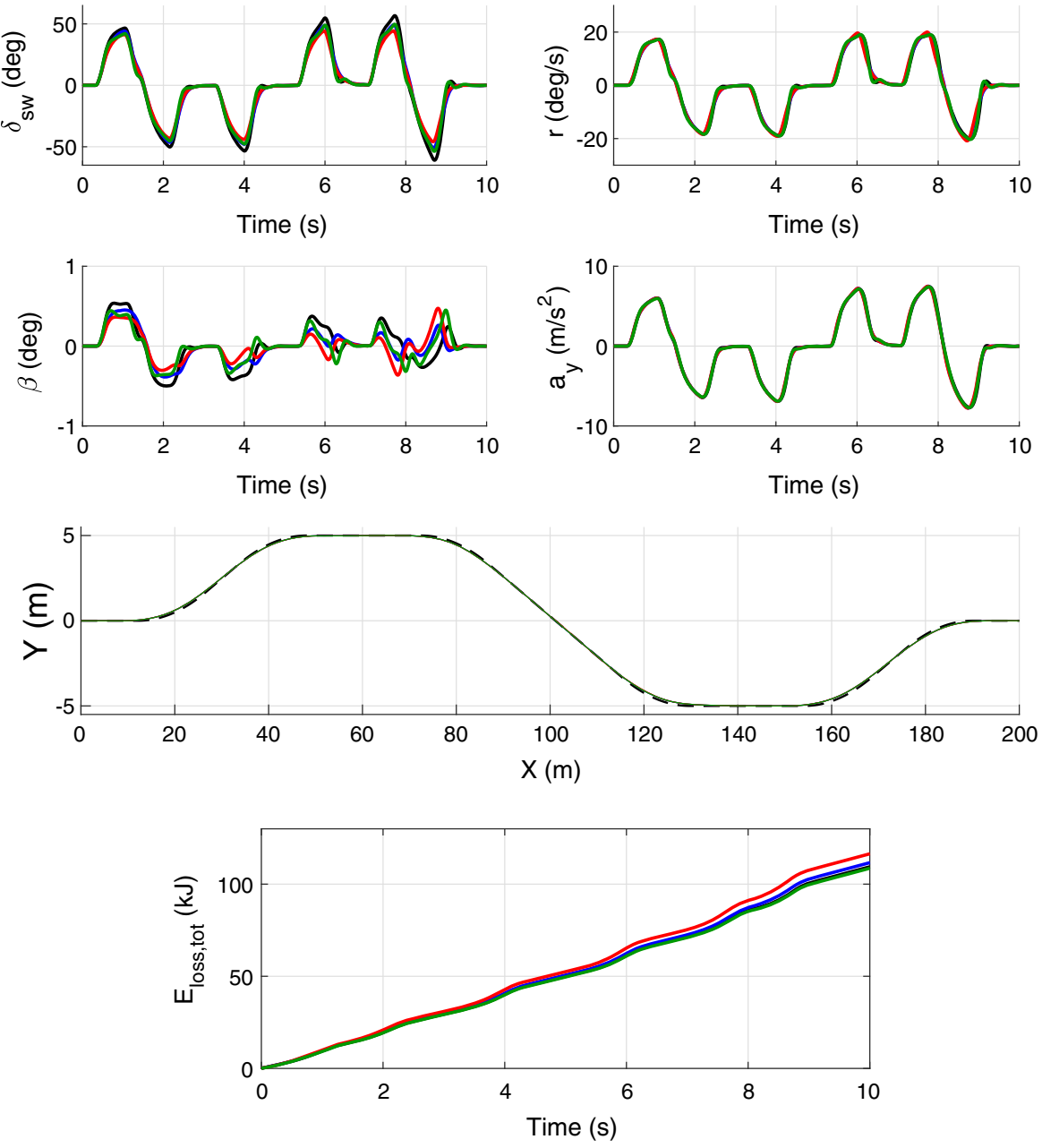

is worth to mention that the order of magnitude of the achieved energy efficiency improvement is in line with experimental results obtained in the literature, e.g., in [23].

\section{Conclusions}

The analysis presented in this paper leads to the following conclusions:

- The developed integrated torque-vectoring framework ensures a smooth cooperation between the three main blocks of a TV controller, and it allows to achieve multiple control objectives at the same time.
- The driving mode selector allows the driver to modify the vehicle behaviour based on his/her preferences, including modes that modify the vehicle cornering response in different ways, or a specific mode that maximises the vehicle energy efficiency.

- The Energy efficiency mode makes the most of the actuation redundancy, focusing solely on minimising the vehicle energy consumption. It should be used together with an appropriate driving style from the driver.

- The high level controller provides a trade-off between yaw rate tracking and sideslip angle tracking, prioritising the latter in safety-critical conditions, and mitigating excessive sideslip angle rates to enhance vehicle safety. 
- The low level controller allows to increase the driving range of the electric vehicle by maximising the vehicle energy efficiency, even when the torque-vectoring controller is mainly used to modify the vehicle handling behaviour.

Future steps include the implementation and assessment of the developed TV framework on an experimental vehicle demonstrator.

Acknowledgements The authors wish to express their gratitude to prof. Giovanni Lutzemberger (University of Pisa, Italy) for his support and for providing the electric motor power loss data.

\section{Compliance with ethical standards}

Conflict of interest The authors declare that they have no conflict of interest.

Open Access This article is licensed under a Creative Commons Attribution 4.0 International License, which permits use, sharing, adaptation, distribution and reproduction in any medium or format, as long as you give appropriate credit to the original author(s) and the source, provide a link to the Creative Commons licence, and indicate if changes were made. The images or other third party material in this article are included in the article's Creative Commons licence, unless indicated otherwise in a credit line to the material. If material is not included in the article's Creative Commons licence and your intended use is not permitted by statutory regulation or exceeds the permitted use, you will need to obtain permission directly from the copyright holder. To view a copy of this licence, visit http://creativecommons.org/licenses/by/4.0/.

\section{Appendix: Vehicle dynamics model derivation for $L Q R$}

A vehicle dynamics model is fully described by three sets of equations [37]: equilibrium, congruence, constitutive.

The equilibrium equations can be obtained through the simplified free-body diagram of the single-track vehicle depicted in Fig. 12.

Specifically, the translational equilibrium along the lateral direction ( $y$, positive to the left) and the rotational equilibrium along the vertical direction $(z$, defined according to the right-hand rule) produce, assuming the steering angle $\delta \approx 0$ :

$$
\begin{aligned}
m a_{y} & =F_{y_{1}}+F_{y_{2}} \\
J_{z} \dot{r} & =F_{y_{1}} a-F_{y_{2}} b+M_{z}
\end{aligned}
$$

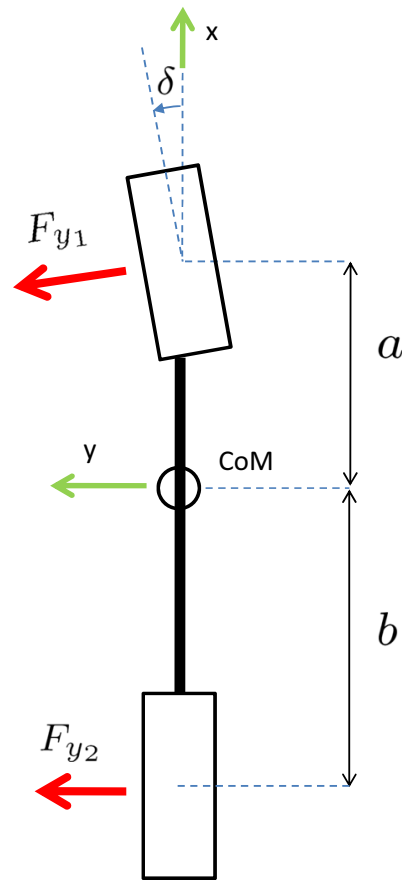

Fig. 12 Free-body diagram of a single-track vehicle model: lateral forces are in red, reference frame axes (centred in the Centre of Mass, $\mathrm{CoM}$ ) are in green. (Color figure online)

where $b$ is the vehicle rear semi-wheelbase, $F_{y_{1}}$ and $F_{y_{2}}$ are the lateral forces at the front and rear axle, respectively. The effect of $M_{z}$ is directly added to the yaw equilibrium equation because of the single-track approximation of the vehicle, as in [56].

Congruence equations are kinematic relationships that relate steering angle, tyre slip angles and vehicle sideslip angle [37].

$$
\begin{aligned}
& \alpha_{1}=\delta-\left(\beta+\frac{r a}{u}\right) \\
& \alpha_{2}=-\left(\beta-\frac{r b}{u}\right)
\end{aligned}
$$

Constitutive equations express the tyre behaviour by relating forces to relevant deformation-related quantities, in this case respectively lateral forces and slip angles. For the purposes of the Linear Quadratic Regulator, the following classical linear relationship $[37,40]$ is used:

$$
\begin{aligned}
& F_{y_{1}}=C_{1} \alpha_{1} \\
& F_{y_{2}}=C_{1} \alpha_{2}
\end{aligned}
$$


By combining equilibrium, congruence and constitutive equations, then using the kinematic relationship 9, considering that $u \approx V$, and finally by collecting terms multiplying respectively $\beta, r$ and $\delta$, one obtains the equations in 11 .

\section{References}

1. Hayes JG, Goodarzi GA (2018) Electric powertrain: energy systems, power electronics and drives for hybrid, electric and fuel cell vehicles. Wiley, New York

2. Kasper R, Schünemann M (2012) 5. Elektrische Fahrantriebe Topologien Und Wirkungsgrad. MTZ-Motortechnische Zeitschrift 73(10):802

3. Jarzebowicz L, Opalinski A (2017) Frequency and time domain characteristics of digital control of electric vehicle in-wheel drives. Arch Electr Eng 66(4):829-842

4. De Novellis L, Sorniotti A, Gruber P (2014) Design and comparison of the handling performance of different electric vehicle layouts. Proc Inst Mech Eng Part D J Automob Eng 228(2):218

5. Siampis E, Velenis E, Longo S (2016) Front-to-rear torque vectoring Model Predictive Control for terminal understeer mitigation. In: The Dynamics of Vehicles on Roads and Tracks: proceedings of the 24th Symposium of the International Association for Vehicle System Dynamics (IAVSD 2015), Graz, Austria, 17-21 August 2015. CRC Press, Cambridge, p 153

6. Esmailzadeh E, Goodarzi A, Vossoughi G (2003) Optimal yaw moment control law for improved vehicle handling. Mechatronics 13(7):659

7. Lenzo B, Gruber P, Sorniotti A (2019) On the enhancement of vehicle handling and energy efficiency of electric vehicles with multiple motors: the iCOMPOSE project. In: The IAVSD international symposium on dynamics of vehicles on roads and tracks. Springer, Berlin, pp 1342-1349

8. Sforza A, Lenzo B, Timpone F (2019) A state-of-the-art review on torque distribution strategies aimed at enhancing energy efficiency for fully electric vehicles with independently actuated drivetrains. Int J Mech Control 20(2):3

9. De Filippis G, Lenzo B, Sorniotti A, Sannen K, De Smet J, Gruber P (2016) On the energy efficiency of electric vehicles with multiple motors. In: 2016 IEEE vehicle power and propulsion conference (VPPC). IEEE, pp 1-6

10. Lenzo B, Sorniotti A, Gruber P, Sannen K (2017) On the experimental analysis of single input single output control of yaw rate and sideslip angle. Int $\mathbf{J}$ Automot Technol 18(5):799

11. Lee K, Li SE, Kum D (2018) Synthesis of robust lane keeping systems: impact of controller and design parameters on system performance. IEEE Trans Intell Transp Syst 20(8):3129

12. Sieber M, Siedersberger KH, Siegel A, Färber B (2015) Automatic emergency steering with distracted drivers: effects of intervention design. In: 2015 IEEE 18th international conference on intelligent transportation systems. IEEE, pp 2040-2045

13. Inoue $\mathrm{S}$, Ozawa $\mathrm{T}$, Inoue $\mathrm{H}$, Raksincharoensak $\mathrm{P}$, Nagai $\mathrm{M}$ (2016) Cooperative lateral control between driver and ADAS by haptic shared control using steering torque assistance combined with direct yaw moment control. In: 2016 IEEE 19th international conference on intelligent transportation systems (ITSC). IEEE, pp 316-321

14. Piyabongkarn D, Lew JY, Rajamani R, Grogg JA (2010) Active driveline torque-management systems. IEEE Control Syst Mag 30(4):86

15. De Novellis L, Sorniotti A, Gruber P (2013) Optimal wheel torque distribution for a four-wheel-drive fully electric vehicle. SAE Int J Passenger Cars Mech Syst 6:128

16. Koehler S, Viehl A, Bringmann O, Rosenstiel W (2015) Improved energy efficiency and vehicle dynamics for battery electric vehicles through torque vectoring control. In: Intelligent vehicles symposium (IV), 2015 IEEE. IEEE, pp 749-754

17. Wong A, Kasinathan D, Khajepour A, Chen SK, Litkouhi B (2016) Integrated torque vectoring and power management framework for electric vehicles. Control Eng Pract 48:22

18. Vignati M, Sabbioni E, Tarsitano D (2016) Torque vectoring control for IWM vehicles. Int J Veh Perform 2(3):302

19. De Novellis L, Sorniotti A, Gruber P (2014) Wheel torque distribution criteria for electric vehicles with torque-vectoring differentials. IEEE Trans Veh Technol 63(4):1593

20. De Novellis L, Sorniotti A, Gruber P (2015) Driving modes for designing the cornering response of fully electric vehicles with multiple motors. Mech Syst Signal Process 64:1

21. Lenzo B, Zanchetta M, Sorniotti A, Gruber P, De Nijs W (2021) Yaw rate and sideslip angle control through single input single output direct yaw moment control. IEEE Trans Control Syst Technol 29:124-139

22. Lenzo B, Sorniotti A, De Filippis G, Gruber P, Sannen K (2016) Understeer characteristics for energy-efficient fully electric vehicles with multiple motors. In: EVS29 international battery, hybrid and fuel cell electric vehicle symposium proceedings

23. De Filippis G, Lenzo B, Sorniotti A, Gruber P, De Nijs W (2018) Energy-efficient torque-vectoring control of electric vehicles with multiple drivetrains. IEEE Trans Veh Technol 67:4702-4715

24. Lu Q, Sorniotti A, Gruber P, Theunissen J, De Smet J (2016) $\mathrm{H}$-infinity loop shaping for the torque-vectoring control of electric vehicles: theoretical design and experimental assessment. Mechatronics 35:32

25. Tota A, Lenzo B, Lu Q, Sorniotti A, Gruber P, Fallah S, Velardocchia M, Galvagno E, De Smet J (2018) On the experimental analysis of integral sliding modes for yaw rate and sideslip control of an electric vehicle with multiple motors. Int J Automot Technol 19(5):811

26. Goggia T, Sorniotti A, De Novellis L, Ferrara A, Gruber P, Theunissen J, Steenbeke D, Knauder B, Zehetner J (2015) Integral sliding mode for the torque-vectoring control of fully electric vehicles: theoretical design and experimental assessment. IEEE Trans Veh Technol 64(5):1701

27. Chen L, Chen T, Xu X, Cai Y, Jiang H, Sun X (2018) MultiObjective coordination control strategy of distributed drive electric vehicle by orientated tire force distribution method. IEEE Access 6:69559 
28. De Novellis L, Sorniotti A, Gruber P, Pennycott A (2014) Comparison of feedback control techniques for torquevectoring control of fully electric vehicles. IEEE Trans Veh Technol 63(8):3612

29. Barbaraci G, Virzi'Mariotti G (2011) Performances comparison for a rotating shaft suspended by 4-axis radial active magnetic bearings via $\mu$-synthesis, loop-shaping design, and sub $(\mathrm{H}) 8$ with uncertainties. Modell Simul Eng 2011:414286

30. Dizqah AM, Lenzo B, Sorniotti A, Gruber P, Fallah S, De Smet J (2016) A fast and parametric torque distribution strategy for four-wheel-drive energy-efficient electric vehicles. IEEE Trans Ind Electron 63(7):4367

31. Parker G, Griffin J, Popov A (2016) The effect on power consumption \& handling of efficiency-driven active torque distribution in a four wheeled vehicle. In: The dynamics of vehicles on roads and tracks: proceedings of the 24th symposium of the international association for vehicle system dynamics (IAVSD 2015), Graz, Austria, 17-21 August 2015, p 97

32. Pennycott A, Novellis LD, Gruber P, Sorniotti A (2015) Sources of power loss during torque-vectoring for fully electric vehicles. Int J Veh Des 67(2):157

33. Lenzo B, De Filippis G, Dizqah AM, Sorniotti A, Gruber P, Fallah S, De Nijs W (2017) Torque distribution strategies for energy-efficient electric vehicles with multiple drivetrains. J Dyn Syst Meas Control 139(12):121004-121013

34. Lin C, Xu Z (2015) Wheel torque distribution of fourwheel-drive electric vehicles based on multi-objective optimization. Energies 8(5):3815

35. Mahmoudi A, Soong WL, Pellegrino G, Armando E (2015) Efficiency maps of electrical machines. In: Energy conversion congress and exposition (ECCE), 2015 IEEE. IEEE, pp 2791-2799

36. Guo H, Cao D, Chen H, Lv C, Wang H, Yang S (2018) Vehicle dynamic state estimation: state of the art schemes and perspectives. IEEE/CAA J Autom Sin 5(2):418

37. Guiggiani M (2018) The science of vehicle dynamics. Springer, Berlin

38. Pacejka H (2012) Tire and vehicle dynamics, 3rd edn. Elsevier Science, New York

39. Sabbioni E, Cheli F, Vignati M, Melzi S (2014) Comparison of torque vectoring control strategies for a IWM vehicle. SAE Int J Passenger Cars-Electronic Electr Syst 7:565

40. Genta G (1997) Motor vehicle dynamics: modeling and simulation, vol 43. World Scientific, Singapore

41. Farroni F, Russo M, Russo R, Terzo M, Timpone F (2013) A combined use of phase plane and handling diagram method to study the influence of tyre and vehicle characteristics on stability. Veh Syst Dyn 51(8):1265

42. Barbaraci G, Mariotti GV (2012) Sub-optimal control law for active magnetic bearings suspension. J Control Eng Technol (JCET) 2(1):1
43. Rajamani R (2011) Vehicle dynamics and control. Springer, Berlin

44. He JB, Wang QG, Lee TH (2000) PI/PID controller tuning via LQR approach. Chem Eng Sci 55(13):2429

45. https://www.bosch-motorsport.com/content/downloads/ Raceparts/Resources/pdf/Data. Accessed 21 Jan 2021

46. https://www.boschrexroth.com/en/au/products/product-groups/ mobile-hydraulics/mobile-electronics/bodas-hardware/bodassensors/mm5-10. Accessed 21 Jan 2021

47. https://www.bosch-mobility-solutions.com/en/products-andservices/passenger-cars-and-light-commercial-vehicles/ driving-safety-systems/electronic-stability-program/inertialsensor. Accessed 21 Jan 2021

48. https://www.boschrexroth.com/en/xc/products/product-groups/ mobile-hydraulics/mobile-electronics/bodas-hardware/bodassensors/dsa. Accessed 21 Jan 2021

49. Kobayashi K, Cheok KC, Watanabe K (1995) Estimation of absolute vehicle speed using fuzzy logic rule-based Kalman filter. In: Proceedings of 1995 American Control Conference-ACC'95, vol. 5. IEEE, pp 3086-3090

50. Daiss A, Kiencke U (1995) Estimation of vehicle speed fuzzy-estimation in comparison with Kalman-filtering. In: Proceedings of international conference on control applications. IEEE, pp 281-284

51. Cheli F, Sabbioni E, Pesce M, Melzi S (2007) A methodology for vehicle sideslip angle identification: comparison with experimental data. Veh Syst Dyn 45(6):549

52. Di Biase F, Lenzo B, Timpone F (2020) Vehicle sideslip angle estimation for a heavy-duty vehicle via Extended Kalman Filter using a Rational tyre model. IEEE Access 8:142120

53. Cheli F, Leo E, Melzi S, Mancosu F (2006) A 14dof model for the evaluation of vehicle's dynamics: numerical-experimental comparison. Int J Mech Control 6(2):19

54. Lenzo B, Bucchi F, Sorniotti A, Frendo F (2019) On the handling performance of a vehicle with different front-torear wheel torque distributions. Veh Syst Dyn 57(11):1685

55. Gruber P, Sorniotti A, Lenzo B, De Filippis G, Fallah S (2016) Energy efficient torque vectoring control. In: Advanced vehicle control AVEC. CRC Press, Cambridge, pp 17-22

56. Lu Q, Gentile P, Tota A, Sorniotti A, Gruber P, Costamagna F, De Smet J (2016) Enhancing vehicle cornering limit through sideslip and yaw rate control. Mech Syst Signal Process 75:455

Publisher's Note Springer Nature remains neutral with regard to jurisdictional claims in published maps and institutional affiliations. 